\title{
Editorial Comment to "Robotic-Assisted Surgery for Upper Tract Urothelial Carcinoma: A Comparative Survival Analysis"
}

\author{
Chawawat Gosrisirikul ${ }^{1}$, Dong Hoon $\mathrm{Koh}^{2}$, and Koon Ho Rha ${ }^{2}$ \\ ${ }^{1}$ Department of Urology, Rajavithi Hospital, Bangkok, Thailand; ${ }^{2}$ Department of Urology, Severance Hospital, Urological \\ Science Institute, Yonsei University College of Medicine, Seoul, Korea
}

In the review article, robotic-assisted laparoscopic (RAL) was utilized in patients undergoing surgery for upper tract urothelial carcinoma compared with open and laparoscopic approaches. In a retrospective analysis, the authors compared survival (overall and cancer-specific) and intravesical recurrence rates based on surgical approach (open, laparoscopic, or RAL). Including 3801 patients (65-85 years) diagnosed with nonmetastatic upper tract urothelial carcinoma (UTUC) undergoing removal of the kidney and/or ureter were identified using Medicarelinked SEER Program data (2004-2013). Open $(n=1862)$, laparoscopic $(n=1624)$, and robotic $(n=315)$.

Primary Outcomes Three-year overall (OS) rates for the RAL, laparoscopic, and open groups were 67, 66, and 63\%, respectively ( $p=0.037)$. However, on univariable analysis, no difference in OS or cancer-specific survival (CSS) was noted. Independent predictors of increased OS on multivariable analysis included younger age $(p<0.001)$, fewer comorbid conditions $(p<0.001)$, and having more $\mathrm{LN}$ removed. The only factor independently predictive of higher CSS was increased number of LN surgically removed. No difference in rate of intravesical recurrence was noted based on operative approach $(p=0.480)$.

Secondary Outcomes Length of hospital stay was shortest in the RAL group $(p<0.001)$ but had the highest in-hospital charges $(p<0.001$ compared with both open and laparoscopic). No significant difference in 30 - or 90-day readmission rates were noted between groups. In this study, subgroup analysis of the radical

(C) Society of Surgical Oncology 2018

First Received: 31 May 2018;

Published Online: 26 June 2018

K. H. Rha

e-mail: Khrha@yuhs.ac nephroureterectomy patients found that $33 \%$ of RAL underwent lymphadenectomy compared with 17 and $21 \%$ in the laparoscopic and open groups, respectively $(p<0.001)$.

Therefore, RAL in UTUC surgery is feasibility to have equivalent survival (OS or CSS) and intravesical recurrence rates compared with those undergoing a laparoscopic or open approach. Also, the RAL technique is associated with an increased likelihood of lymphadenectomy, which might improve survival.

Subsequent studies should further classify the ureteral tumor location (proximal, mid, distal), which might be the confounding factor risk of intravesical recurrence, including younger patients, more numbers of the RAL group in the evolving robotic era, and the role of robotic segmental ureterectomy in the part of ureteral cancer in high medical comorbidity patients compared with standard radical nephroureterectomy or compared with open and laparoscopic approaches. ${ }^{1-3}$ Prospective, randomized trials should be conducted in single or multi-institutional series. ${ }^{4}$ Further studies are needed to validate the importance of lymphadenectomy in UTUC. ${ }^{5}$ Despite available studies evaluating templates to date, it is not possible to standardise indication or extent of lymphadenectomy. ${ }^{6}$

\section{REFERENCES}

1. Aboumohamed AA, Krane LS, Hemal AK. Oncologic outcomes following robot-assisted laparoscopic nephroureterectomy with bladder cuff excision for upper tract urothelial carcinoma. J Urol. 2015;194(6):1561-6.

2. Rodriguez JF, et al. Utilization and outcomes of nephroureterectomy for upper tract urothelial carcinoma by surgical approach. $J$ Endourol. 2017;31(7):661-5.

3. Chang KD, Raheem AA, Rha KH. Novel robotic systems and future directions. Indian J Urol. 2018;34(2):110. 
4. Raheem AA, et al. Feasibility of robot-assisted segmental ureterectomy and ureteroureterostomy in patient with high medical comorbidity. Int Braz J Urol. 2017;43:779-80.

5. Moschini M, et al. Trends of lymphadenectomy in upper tract urothelial carcinoma (UTUC) patients treated with radical nephroureterectomy. World J Urol. 2017;35(10):1541-7.
6. Roscigno M, et al. Lymphadenectomy at the time of nephroureterectomy for upper tract urothelial cancer. Eur Urol. 2011;60(4): 776-83. 